\title{
The Yo-Yo tests and twelve-minute run test performance in young Japanese cross-country skiers
}

\author{
Denda Yuki, Oh Taewoong, Nakajima Setsuko \\ Graduate School of Health Science, Matsumoto University, Matsumoto, Japan; ${ }^{*}$ Corresponding Author: taewoong@matsu.ac.jp \\ Received 17 January 2013; revised 14 March 2013; accepted 17 April 2013 \\ Copyright (C) 2013 Denda Yuki et al. This is an open access article distributed under the Creative Commons Attribution License, \\ which permits unrestricted use, distribution, and reproduction in any medium, provided the original work is properly cited.
}

\begin{abstract}
Purpose: The aim of the present study is to examine the performance of young Japanese cross-country skiers of different competitive levels with the use of Yo-Yo tests and the 12minute run test (12MRT) that have been demonstrated to be related to each level, and to know which test is suitable for different competitive performance. Methods: Elite group $(\mathrm{N}=9)$ was $16.9 \pm 0.3$ years and moderate trained group $(\mathrm{N}=$ 22) was $16.1 \pm 0.9$ years. The testing sessions consisted of the following: 1) Vertical jump and $50 \mathrm{~m}$ sprint; 2) the Yo-Yo tests level 1 and 2 (intermittent recovery); 3) 12MRT. Results: Results showed the vertical jump heights and $50 \mathrm{~m}$ sprint that no significant both performances differences were found among the two groups. Significant the Yo-Yo tests and 12MRT performances differences were found between the two groups $(p<0.05)$. Performance test $(\%)$ showed the ratio of moderate trained group towards elite group. The Yo-Yo tests performance was low enough compared with the result of 12MRT in moderate trained group. Conclusions: It is therefore valid to assume that low Yo-Yo tests performance due to immature anaerobic in crosscountry skiers was a new limiting factor in the competitive level.
\end{abstract}

Keywords: Yo-Yo Intermittent Recovery Tests; Endurance Sports; Aerobic; Anaerobic;

Performance

\section{INTRODUCTION}

Cross-country skiing is known for having the longest game time and distance in winter sports, and also a typical endurance sport. The competitive event of the cross- country skiing ranges from 5-kilometre races to a long endurance that is about 50 kilometers (racing times from $25 \mathrm{~min}$ to $3 \mathrm{~h}$ [1] or more [2]). This sport demands high aerobic capacity and according to some studies, the maximal oxygen uptake is more than $80 \mathrm{ml} / \mathrm{kg} / \mathrm{min}$ [3]. Thus, aerobic capacity is one of the most important factors in performing cross-country skiing [2].

However, the new appraisal of the performance factor in modern cross-country skiing is the shorter sprint. Previous study investigated the physiological training in world-class and national-level sprint cross-country skiers regarding physiological responses, $\mathrm{VO}_{2}$ peak and incremental peak treadmill speed test in world-class group is $8 \%$ higher than national-level. The current results show that world-class skiers have superior aerobic capacity, efficiency, and high-speed capacity and it is suggested that these variables determine sprint skiing performance [1]. In addition, racing times leading to higher average speeds, the need for acceleration in the start and a high maximal speed in the finish have increased the importance of high speed capacities in cross-country skiing in races [1]. Patricia [2] says that, in any race where no more than a few seconds separate the top finishers, the rate of anaerobic glycolysis, i.e. anaerobic power, can account for the difference between winning and losing. Cross-country skiing performance should have anaerobic capacity as well as aerobic capacity. Thus, cross-country ski has aerobic and anaerobic capacities of incorporating the sport.

Traditionally, cross-country skiing has been characterized as maximal oxygen uptake of treadmill running [4] and ski-walking [1,5] in aerobic performance tests, and anaerobic capacity of the Margaria-Kalamen test, vertical jump tests [2] and maximal isometric one-leg strength test [1]. These tests are not always display explosive power in aerobic of constant pace, but except in fixed position. However, the aerobic loading is high throughout the competition and the anaerobic energy turnover is extensive during the race. The Yo-Yo tests are used recently to 
measure the aerobic-anaerobic capacity rather than the twelve-minute run test (12MRT), which is typical endurance measuring method. Recent studies have demonstrated that the Yo-Yo intermittent recovery test is related to physical performance throughout the match of elite soccer referees [6,7] and the 12MRT performance [8]. In addition, it is possible to measure speed characteristic endurance of players in a game [9]. It is also important to measure aerobic and anaerobic behavior for cross-country skiers with only one test. No research that measures the endurance of the explosive power in the aerobic. It is clear that the Yo-Yo tests are necessary in intermittent sports, but research for the endurance sports haven't done yet.

The aim of the present study is to examine the performance of young Japanese cross-country skiers of different competitive levels with the use of Yo-Yo tests [8] and the 12MRT that have been demonstrated to be related to each level, and to know which test is suitable for different competitive performance.

\section{METHODS}

\subsection{Experimental Approach to the Problem}

In the present investigation, a cross-sectional approach to examine the performance of cross-country skiers of different competitive levels was by the two field tests and two physical tests. Subjects were categorized in elite and moderate trained group. Elite group competed in national athletic meet in high school while moderate trained group did not. The testing sessions consisted of the following: 1) Vertical jump and $50 \mathrm{~m}$ sprint; 2) the Yo-Yo tests level 1 and 2 (Intermittent Recovery); 3) 12MRT. The subjects' blood lactates were examined after the endurance tests.

\subsection{Subjects}

Participants were thirty-one active male high school cross-country skiers in Nagano, Japan who competed in the 2010-2011 competitive season. All players had at least 5 years of experience in competitive cross-country skiing. Subjects have been practicing five days a week and more than three hours a day. Age, height, and weight of elite group $(\mathrm{N}=9)$ were $16.9 \pm 0.3$ years, $170.7 \pm 4.5$ $\mathrm{cm}$, and $62.4 \pm 7.0 \mathrm{~kg}$, moderate trained group $(\mathrm{N}=22)$ were $16.1 \pm 0.9$ years, $168.2 \pm 6.6 \mathrm{~cm}$, and $63.0 \pm 9.0 \mathrm{~kg}$. Subjects were fully informed of all experimental procedures and risks before giving their written informed consent to participate. The Ethics Committee of Matsumoto University, Japan approved the study.

\subsection{Testing Procedures}

Testing sessions consisted of vertical jump, Yo-Yo tests performed indoor on a wooden surface, $50 \mathrm{~m}$ sprint and 12MRT performed outdoor on a ground during autumn of year 2011. On the test day, two hours before the testing sessions only water is allowed, and subjects must done consuming a light meal at least two hours before the beginning of exercise. Each player was instructed and verbally encouraged to give their fullest effort during all tests. A standardized warm-up, consisting of jogging and a series of increasing intensity sprints, was performed prior to the test. The pre-experiment session took one week before the commencement of the experimental procedures. Testing sessions took 1-week intervals.

Vertical jump and $50 \mathrm{~m}$ sprint tests were done to assess the explosive power and speed performances. Vertical jump was measured using a measurement board (SENOH, LC9011, Japan). Subjects were asked to keep their hands on hips to prevent the influence of arm movements on the test [10]. Each subject performed at least two maximum vertical jumps starting from a standing position, with one minute of recovery time. The highest jump was used for calculations [11]. $50 \mathrm{~m}$ sprint test were measured using a hand-held stopwatch (SEIKO, S056-4000, Japan). Time was recorded accurately to the nearest 0.01 seconds.

This study was also tested in the Yo-Yo Intermittent Recovery Test (YYIRT) level 1 and 2, respectively. The Yo-Yo tests were performed according to the guidelines established by Bangsbo [12]. YYIRT has 2 levels differing with respect to starting speed [9]. YYIRT can be regarded as an aerobic-anaerobic [13,14], intermittentspecific field test. Krustrup [14] says that when comparing the performance of players from an elite team with players at lower levels, it was also clear that elite players performed better on the Yo-Yo IRT2.

Aerobic capacity in maximal oxygen intake the use of the $12 \mathrm{MRT}$, reported that this test was more related with the increased treadmill test [15].

\subsection{Blood Analysis}

Immediately after the end of the Yo-Yo tests and the 12MRT, blood samples were taken from the fingertips for blood lactate concentration (BLC) analysis. Pretest BLC was taken immediately after the end of a self-administered warm-up [8]. BLC was determined using a portable lactate analyzer (Lactate PRO, Arkray Inc., Kyoto, Japan). Validity and reliability of Lactate Pro respect to the Yo-Yo tests and 12MRT have been reported [8].

\subsection{Statistical Analysis}

All measurement results were presented as mean $\pm \mathrm{SD}$. Data analysis were performed using unpaired $\mathrm{T}$ test to determine significant differences in elite group and mod- 
erate trained group results. Pearson's correlation coefficients have been used to examine possible relationships between variables. Differences between the tests in delta values were evaluated by one-way ANOVA, in BLC and also to the percentage performance test. When a significant main effect was detected, data were subsequently analyzed using a Tukey post hoc test. Significance was set at $\mathrm{p}<0.05$, respectively. SPSS version 19 for Windows was used for all statistical analysis.

\section{RESULTS}

All participants $(\mathrm{N}=31)$ elite group $(\mathrm{N}=9)$ and moderate trained group $(\mathrm{N}=22)$, which the Yo-Yo tests and 12MRT performances were presented in Table 1. YYIRT1 and YYIRT2, 12MRT performances' significant differences were found between elite and moderate trained groups $(\mathrm{p}<0.05)$. Explosive power and speed performances were also presented in Table 1. No significant differences were found between the two groups, respectively. Figure 1 presented that moderate trained group was lower compared to elite group in the test. There was a correlation between 12MRT significant to YYIRT1 $(\mathrm{N}=22, \mathrm{r}=0.81, \mathrm{p}<0.01)$ and to YYIRT2 $(\mathrm{N}$ $=22, \mathrm{r}=0.61, \mathrm{p}<0.01)$, respectively. BLC of the Yo-Yo tests and 12MRT were YYIRT1; $14.6 \pm 3.9$ and $13.5 \pm$ $4.2 \mathrm{mmol} \cdot \mathrm{L}^{-1}(\mathrm{p}>0.05)$, YYIRT2; $15.5 \pm 3.1$ and $15.5 \pm$ $3.9 \mathrm{mmol} \cdot \mathrm{L}^{-1}(\mathrm{p}>0.05), 12 \mathrm{MRT} ; 11.9 \pm 3.9$ and $12.0 \pm$ $3.0 \mathrm{mmol} \cdot \mathrm{L}^{-1}(\mathrm{p}>0.05)$, for elite and moderate trained group, respectively. After the test, each BLC has no significant differences between the two groups. Before the test occurred the BLCs' of two groups were incomparable or almost has the same BLC. BLC differences between the Yo-Yo tests and 12MRT were presented in Figure 2.

\section{DISCUSSION}

Traditionally, continuous endurance test was used in the assessment of competitive aerobic performance $[1,3$, 16]. This study reported $12 \mathrm{MRT}$ was more related with $\mathrm{VO}_{2} \max$ [15] and the competitive levels of cross-country skiing differences were shown. As in the past, elite cross-country skiers demonstrated in 12MRT that aerobic power is important ${ }^{2}$. Another finding, the explosive power and speed performances have no significant differences between the two groups. Present study marked in the occurred YYIRT1 and YYIRT2 performances' significant differences between the two groups. Yo-Yo

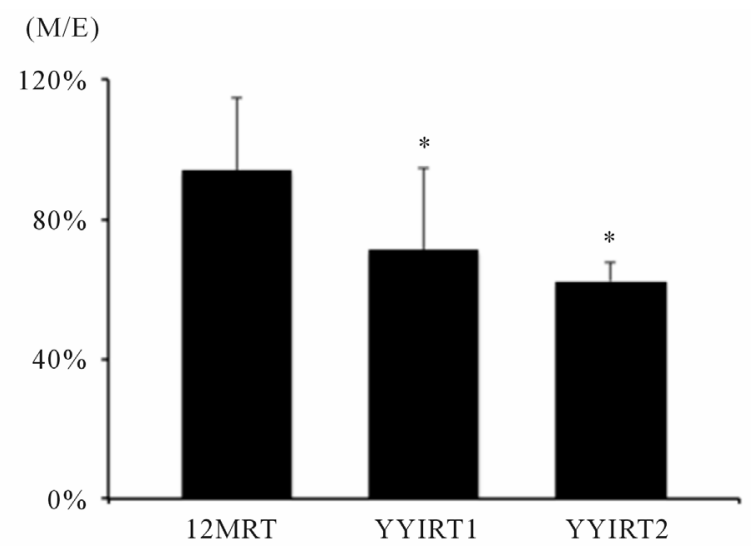

Figure 1. Performance test $(\%)$ showed the ratio of moderate trained group towards elite group. 12MRT (94\%, $\mathrm{N}=17)$, YYIRT1 $(71 \%, \mathrm{~N}=18)$, and YYIRT2 $(62 \%, \mathrm{~N}=20) . \mathrm{M} / \mathrm{E}$ : moderate trained group/elite group. ${ }^{*}$ Significantly different from 12MRT $(\mathrm{p}<0.01)$.

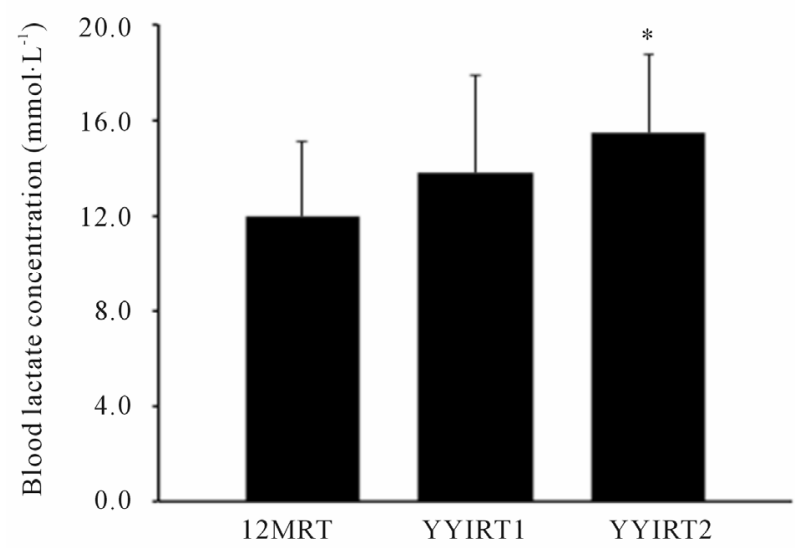

Figure 2. BLC differences between the Yo-Yo tests and 12MRT. 12MRT $(\mathrm{N}=24)$, YYIRT1 $(\mathrm{N}=25)$, and YYIRT2 $(\mathrm{N}=25)$. *Significantly different from 12MRT $(\mathrm{p}<0.05)$.

Table 1. Test performances in young Japanese cross-country skiers.

\begin{tabular}{|c|c|c|c|c|}
\hline Performances & $\mathrm{N}$ & All & Moderate trained group & Elite group \\
\hline 50 m Sprint (sec) & $\mathrm{E}=7, \mathrm{M}=15$ & $7.22 \pm 0.47$ & $7.25 \pm 0.53$ & $7.14 \pm 0.28$ \\
\hline YYIRT1 (m) & $\mathrm{E}=8, \mathrm{M}=18$ & $1746 \pm 576$ & $1553 \pm 451$ & $2180 \pm 617^{* *}$ \\
\hline YYIRT2 (m) & $\mathrm{E}=8, \mathrm{M}=20$ & $521 \pm 233$ & $444 \pm 168$ & $715 \pm 271^{* *}$ \\
\hline 12MRT (m) & $\mathrm{E}=7, \mathrm{M}=17$ & $3093 \pm 205$ & $3037 \pm 190$ & $3230 \pm 182^{*}$ \\
\hline
\end{tabular}

Significantly different from moderate trained level values, ${ }^{*} \mathrm{p}<0.05 ;{ }^{* *} \mathrm{p}<0.01$. E: elite group, M: moderate trained group. 
tests were the specific test for intermittent sports but it also applicable to endurance sports since the Yo-Yo tests' results were similar to that of intermittent sports. Figure 1 showed Yo-Yo tests were more important than the 12MRT in competitive level of cross-country skiers. Performance test $(\%)$ showed the ratio of moderate trained group towards elite group. The Yo-Yo tests performance was low enough compared with the result of 12MRT in moderate trained group. Consequently, player that has low competitive level was decided by the endurance of anaerobic performance than aerobic performance.

The results discussed that the Yo-Yo tests should be used in preference to the 12MRT because they provide requirement of physical capacity, aerobic and endurance of anaerobic in cross-country skiing. The Yo-Yo tests can discriminate different levels of cross-country skiers. A possible reason for this is exhaustion in both YYIRT1 and YYIRT2 might be reached before maximal activation of the aerobic energy system in moderate trained group having lower anaerobic fitness [17]. In brief, endurance of anaerobic power is emphasized from maximal anaerobic power in cross-country skiers.

Therefore, there were findings obtained that correlation between 12MRT and YYIRT1, YYIRT2. Reported in YYIRT1 $(\mathrm{r}=0.71 ; \mathrm{p}<0.05)$ [18], YYIRT2 $(\mathrm{r}=0.56 ; \mathrm{p}<$ 0.05) [14] were related with $\mathrm{VO}_{2} \max$. These studies proved this finding; accordingly, there was validity of aerobic index in YYIRT1 and YYIRT2.

For another finding of this study was the comparison of the BLC variables in competitive level and among the tests. The Yo-Yo tests and 12MRT has no significant differences in BLC between the groups. A reduction in BLC at a given running speed or work rate is commonly used to indicate increased economy and/or performance [8]. Present study, elite group has better performances than moderate trained group in the Yo-Yo tests and 12MRT because elite group has increased economy of aerobic and anaerobic in exhaustion. YYIRT1 is mainly focusing on an individual's endurance capacity [9], and YYIRT1's anaerobic intensity was lower than YYIRT2 that made the distance to be longer. YYIRT1 performance was possible to run until a lot of blood lactate acids accumulated as well as in 12MRT presented in Figure 2. On the other hand, YYIRT2 has a significant difference in BLC compared to 12MRT. In general, the rate of anaerobic energy production and specifically the rate of lactate production the YYIRT2 were high [9]. YYIRT2 can be related to anaerobic power from the physiological aspect.

Consequently, YYIRT2 can be assessing anaerobic power better than 12MRT. Especially, YYIRT2 can discriminate competitive performance level in physiological and test performance aspects more than 12MRT in competitive level cross-country skiers.

\section{CONCLUSIONS}

Aerobic power of 12MRT performance has the basics of endurance sports. By this study, the Yo-Yo tests had shown its necessity for cross-country skiing performance. Additionally, it revealed as a suitable test for assessing competitive level in young Japanese cross-country skiers. Competitive level difference in the Yo-Yo tests performance expressed conspicuously. It is therefore valid to assume that low Yo-Yo tests performance due to immature anaerobic in cross-country skiers was a new limiting factor in the competitive level. Expressly, YYIRT2 can be considered to distinguish the differences of competitive level of young Japanese cross-country skiers.

From the gathered data, aerobic as the basic requirement and high anaerobic endurance was the requirement to become a top-level cross-country skier, and a useful tool in talent selection for young Japanese cross-country skiers in endurance sports.

\section{REFERENCES}

[1] Sandbakk, O., Holmberg, H.-C., Leirdal, S. and Ettema, G. (2011) The physiology of world-class sprint skiers. Scandinavian Journal of Medicine \& Science in Sports, 21, e9-e16. doi:10.1111/j.1600-0838.2010.01117.x

[2] Patricia, A.E., Johnson, S.C., Bainbridge, C.N. and Zupan, M.F. (1989) Applied physiology of cross-country skiing. Sports Medicine, 8, 67-79. doi:10.2165/00007256-198908020-00001

[3] Rusko, H., Have, M. and Karvinen, E. (1978) Aerobic performance capacity in athletes. European Journal of Applied Physiology and Occupational Physiology, 38, 151-159. doi:10.1007/BF00421531

[4] Snadbakk, O., Welde, B. and Holmberg, H.C. (2011) Endurance training and sprint performance in elite junior cross-country skiers. Journal of Strength \& Conditioning Research, 25, 1229-1305.

[5] Mikkola, J.S., Rusko, H.K. and Nummela, A.T., Paavolainen, L.M. and Hakkinen, K. (2007) Concurrent endurance and explosive type strength training increases activation and fast force production of leg extensor muscles in endurance athletes. Journal of Strength \& Conditioning Research, 21, 613-620.

[6] Castagna, C., Abt, G. and D'Ottavio, S. (2002) Relation between fitness tests and match performance in elite Italian soccer referees. Journal of Strength \& Conditioning Research, 16, 231-235.

[7] Krustrup, P. and Bangsbo, J. (2001) Physiological demands of top-class soccer refereeing in relation to physical capacity: Effect of intense intermittent exercise training. Journal of Sports Science, 19, 881-891. doi: $10.1080 / 026404101753113831$

[8] Castagna C, Abt, G. and D'Ottavio, S. (2005) Competitive-level differences in Yo-Yo intermittent recovery test and twelve minute run test performance in soccer referees. Journal of Strength \& Conditioning Research, 19, 805- 
809.

[9] Bangsbo, J., Iaia, F.M. and Krustrup, P. (2008) The Yo-Yo intermittent recovery test: A useful tool for evaluation of physical performance in intermittent sports. Sports Medicine, 38, 37-51. doi:10.2165/00007256-200838010-00004

[10] Bosco, C., Luhtanen, P. and Komi, P.V. (1983) A simple method for measurement of mechanical power in jumping. European Journal of Applied Physiology and Occupational Physiology, 50, 273-282. doi:10.1007/BF00422166

[11] Castagna, C., Impellizzeri, F.M., Rampinini, E., D’Ottavio, S. and Manzi, V. (2008) The Yo-Yo intermittent recovery test in basketball players. Journal of Science and Medicine in Sport, 11, 202-208. doi:10.1016/j.jsams.2007.02.013

[12] Bangsbo, J. (1996) Yo-Yo test. Kells, Ancona.

[13] Castagna, C., Impellizzeri, F.M., Chamari, K., Carlomagno, D. and Rampinini, E. (2006) Aerobic fitness and Yo-Yo continuous and intermittent tests performances in soccer players: A correlation study. Journal of Strength \& Conditioning Research, 20, 320-325.

[14] Krustrup, P., Mohr, M., Nybo, L., Jensen, J.M., Nielsen, J.J. and Bangsbo, J. (2006) The Yo-Yo IR2 test: Physiological response, reliability, and application to elite soccer.
Medicine and Science in Sports and Exercise, 38, 16661673. doi:10.1249/01.mss.0000227538.20799.08

[15] Cooper, K.H. (1968) A means of assessing maximal oxygen intake. Correlation between field and treadmill testing. Journal of American Medical Association, 203, 201204. doi:10.1001/jama.1968.03140030033008

[16] Metaxas, T.I., Koutlianos, N.A., Kouidi, E.J. and Deligiannis, A.P. (2005) Comparative study of field and laboratory tests for the evaluation of aerobic capacity in soccer players. Journal of Strength \& Conditioning Research, 19, 7984.

[17] Kentaro, C., Yoshihiro, H., Tomomi, I. and Takahiko, N. (2011) Relationships between Yo-Yo intermittent recovery tests and development of aerobic and anaerobic fitness in U-13 and U-17 soccer players. International Journal of Sport and Health Science, 9, 91-97. doi:10.5432/ijshs. 201110

[18] Krustrup, P., Mohr, M., Amstrup, T., Rysgaard, T., Johansen, J., Steensberg, A., Pedersen, P.K. and Bangsbo, J. (2003) The Yo-Yo intermittent recovery test: Physiological response, reliability, and validity. Medicine and Science in Sports and Exercise, 35, 697-705. doi:10.1249/01.MSS.0000058441.94520.32 